\title{
Prediction and Validation of Three Cross Hybrids in Maize (Zea mays L.)
}

\author{
R. Pavan", E. Gangappa, S. Ramesh, A. Mohan Rao and Shailaja Hittalmani \\ Department of Genetics and Plant Breeding, University of Agricultural Sciences, \\ Bangalore -65, Karnataka, India
}

*Corresponding author

\section{A B S T R A C T}

The experiment comprised of six $\mathrm{F}_{1}$ 's, which were selected based on the combinations of low yield $\times$ high yield and low ASI $\times$ high ASI. The selected six $F_{1}$ 's were crossed in full

\section{Keywords}

ASI, Epistasis, Predicted, Realised, TWC

Article Info

Accepted:

12 December 2017

Available Online:

10 January 2018 diallel mating design to synthesis 18 three way crosses was estimated and validated on the basis of performance of their constituent single crosses. Out of 18 hybrids, TWC 3 recorded lower days to tasseling and silking and hence it can be used further in breeding programme. While, TWC 4 (0.32), TWC 11 (0.5), TWC 9 (0.76), TWC 10 (0.79), TWC 14 (0.81) and TWC 5 (0.89) recorded least ASI. Hence, it is suggested to be used in deriving superior inbreds of narrow ASI or hybrids suitable for water deficit conditions. The expected yield performance of TWC 1 (128.13) and TWC 2 (126.24) out yielded best check, Nithyashree (126.00) while, TWC 15 (125.49), TWC 17 (123.19), TWC 16 (121.92), TWC 4 (119.51) out yielded best check, Hema (119.00). All 18 three way crosses manifested significant differences between realised and predicted performance suggesting involvement of epistasis in genetic control of grain yield plant ${ }^{-1}$. By and large, there was a good agreement between realized and predicted performance of three way cross hybrids for all characters except for grain yield plant ${ }^{-1}$ and shelling percentage.

\section{Introduction}

The breakthrough of heterosis phenomenon in the development of hybrids which successfully exploited commercial heterosis in maize is considered as significant milestone in the agriculture history during the present century. During 1908 and 1911, Shull exploited heterosis in maize by developing single cross hybrids by a hybridizing two pure homozygous inbred lines derived from an open-pollinated varieties. However, parental homozygous inbred lines derived from the open-pollinated cultivars were so weak that it was not feasible to use them in commercial hybrid seed production. Consequently, instead of single crosses, three way cross hybrids resulting from the cross between single crosses hybrid and homozygous line were proposed by Jones (1918) as they were more productive than homozygous inbred lines. Subsequently, three-way cross hybrids were also developed as a supplement to double cross hybrids and were grown by farmers during the 1930`s (Crabb, 1992).

Synthesis and identification of heterotic three way cross (TWC) depends on a number of 
single-cross hybrids ( $\mathrm{SCH}$ ) involved in the crosses which become unfeasible with an increase in the number of single-cross hybrids. For instance, with just ten inbred lines the breeders need to develop 630 three way cross hybrids. The performance evaluation of such a large number of three way cross hybrids is a highly resource demanding uphill task. To save the precious resources and time, Jenkins (1934) suggested models to predict the three way cross hybrid performance based on the performance of single crosses. Anderson (1938) found close correspondence between predicted and realized yield of three way cross hybrids in maize. Hence, the present study was carried out to predict and validate the performance of three way cross hybrids in maize.

\section{Materials and Methods}

The experiment comprised of six newly developed $F_{1}$ 's during summer 2012, which were selected based on the combinations of low yield $\times$ high yield and low ASI $\times$ high ASI as detailed below (Table 1). The same six $\mathrm{F}_{1}$ 's were crossed to three inbred lines viz., CML-439, DMRN-21 and CML-139 which were used as males to synthesise 18 TWC hybrids during kharif 2012 at K-block of the Department of Genetics and Plant Breeding, UAS, GKVK, Bangalore. The experiment consisting of 18 three way cross hybrids along with two checks viz., Nithyashree and Hema (public bred hybrids) were evaluated in farmer's field at Sabbenahalli, Chickballapur district (Zone5), Karnataka during rabi 2012 and summer 2013. The experiment was laid out in Randomized Complete Block Design (RCBD) with three replications. Each entry was planted in three rows of $3 \mathrm{~m}$ length with row-to-row and plant-to-plant distance of $60 \mathrm{~cm}$ and $30 \mathrm{~cm}$, respectively. All the recommended package of practices was followed to raise a good and healthy crop under protected condition. Data on 12 different quantitative characters were recorded on 24 randomly selected plants to calculate weights.

Trait means and variances of 13 quantitative traits for each three way cross hybrid were computed as detailed below. These means and variances were estimated as follows

Mean $=\frac{\sum \mathrm{xi}}{\mathrm{n}}$

Variance $=\frac{\sum \mathrm{x}_{\mathrm{i}}^{2}-\frac{\sum \mathrm{xi}}{\mathrm{n}}}{\mathrm{n}-1}$

Variance of sample mean $=$ Variance $/ \mathrm{n}$

Standard error $(\mathrm{SE})=\sqrt{\frac{\text { Variance of TWCDC hybrids population }}{\mathrm{n}}}$

Where,

$\mathrm{x}_{\mathrm{i}}=$ trait value of $\mathrm{i}^{\text {th }}$ plant in TWC/DC hybrids

$\mathrm{n}=$ Number of plants on which data were recorded

Predicting the performances of double cross hybrid

Quantitative trait mean of a TWC $[(\mathrm{A} \times \mathrm{B}) \times \mathrm{C}]$ hybrid was predicted by the average performance of non-parental single crosses hybrids involved in TWC hybrids as parents proposed by Jenkins (1934).

$\overline{(\mathrm{A} \times \mathrm{B}) \times \mathrm{C}}=\frac{(\mathrm{A} \times \mathrm{C})+(\mathrm{B} \times \mathrm{C})}{2}$

The agreement of predicted quantitative trait mean of each of three way cross hybrids with those of observed means was tested using $\chi^{2}$ test. As three way cross hybrid means are not estimated with equal precision, weights 
defined as the reciprocal of trait variances of each of three way cross hybrids were used for calculating $\chi^{2}$ test statistic.

Calculated $\chi^{2}=(\mathrm{O}-\mathrm{E})^{2} \times$ Weight

Where, Weight $=\frac{n}{\sigma_{T W C / D C}^{2}}$

Significance of chi square statistic suggested the non-agreement of predicted and realized trait means of three way cross hybrids. Further, non-agreement also suggested possible involvement of epistasis in controlling the inheritance of 12 traits investigated in the present study.

\section{Results and Discussion}

The per se performance of 18 three way cross hybrids was estimated on the basis of performance of their constituent single crosses. The predicted performance of yield and its component traits of 18 three way crosses over two seasons were presented in Table 2. The mean performance for days to tasseling varied from 57.21 days (TWC 3) to 70.13 days (TWC 4) and for silking it varied from 59.59 days (TWC 3) and 72.04 days (TWC 4). Out of 18 hybrids, TWC 3 recorded lower days to tasseling and silking and hence it can be used further in breeding programme. ASI is concerned, the TWC 4 (0.32) and TWC 1 (1.58) recorded smallest and the highest ASI. The three way cross hybrids, TWC 4 (0.32), TWC 11 (0.5), TWC 9 (0.76), TWC 10 (0.79), TWC 14 (0.81) and TWC 5 (0.89) recorded least ASI. Hence, it is suggested to be used in deriving superior inbreds of narrow ASI or hybrids suitable for water deficit conditions.

The mean performance for yield attributing characters viz., ear length, ear circumference, kernels row $^{-1}$, kernel rows ear ${ }^{-1}, 100$ grain weight $(\mathrm{g})$ and shelling percentage varied from $15.29 \mathrm{~cm}$ (TWC 9) to $17.26 \mathrm{~cm}$ (TWC 1), $13.81 \mathrm{~cm}$ (TWC 14) to $15.02 \mathrm{~cm}$ (TWC 1), 29.06 (TWC 9) to 35.43 (TWC 1), 15.76 (TWC 1) to 14.31 (TWC 18), $24.34 \mathrm{~g}$ (TWC 14) to $29.11 \mathrm{~g}$ (TWC 18) and $82.90 \%$ (TWC 10) to $89.70 \%$ (TWC 7), respectively.

The expected yield performance of three way cross hybrid varied from $102.79 \mathrm{~g}$ (TWC 3) to $128.13 \mathrm{~g}$ (TWC 1). Out of 18 three way crosses, TWC 1 (128.13) and TWC 2 (126.24) out yielded best check, Nithyashree (126.00) while, TWC 15 (125.49), TWC 17 (123.19), TWC 16 (121.92), TWC 4 (119.51) out yielded best check, Hema (119.00)

Table.1 Details of six $F_{1}$ 's selected for synthesis of double cross hybrids

\begin{tabular}{|c|c|c|c|}
\hline SI. No & F1's & \multicolumn{2}{|c|}{ Salient characteristics } \\
\hline 1. & HKI 26-2-4(1-2) × CML 41 & $\begin{array}{l}\text { Low yielding } \\
(0.074 \mathrm{~kg} / \text { plant })\end{array}$ & $\begin{array}{l}\text { High yielding } \\
(0.22 \mathrm{~kg} / \text { plant })\end{array}$ \\
\hline 2. & CML $470-$ Bx $15 \times$ CML 41 & $\begin{array}{l}\text { Low yielding } \\
\text { (0.13 kg/plant) }\end{array}$ & $\begin{array}{l}\text { High yielding } \\
(0.22 \mathrm{~kg} / \text { plant })\end{array}$ \\
\hline 3. & HKI 26-2-4(1-2) × CM 500 & $\begin{array}{l}\text { Low yielding } \\
(0.074 \mathrm{~kg} / \text { plant })\end{array}$ & $\begin{array}{l}\text { High yielding } \\
(0.14 \mathrm{~kg} / \text { plant })\end{array}$ \\
\hline 4. & CML359 × CML 326 & $\begin{array}{l}\text { Low ASI } \\
\text { (2.5 days) }\end{array}$ & $\begin{array}{l}\text { High ASI } \\
\text { (7.7 days) }\end{array}$ \\
\hline 5. & HKI 26-2-4(1-2) × CML 358 & $\begin{array}{l}\text { High ASI } \\
\text { (7.7 days) }\end{array}$ & $\begin{array}{l}\text { Low ASI } \\
\text { (2.0 days) }\end{array}$ \\
\hline 6. & CML $326 \times$ DMRN-21 & $\begin{array}{l}\text { High ASI } \\
\text { (7.7 days) }\end{array}$ & $\begin{array}{c}\text { Low ASI } \\
\text { (1.95days) }\end{array}$ \\
\hline
\end{tabular}


Table. 2 Average performance of 18 three way cross hybrids for 12 quantitative traits in maize over two seasons

\begin{tabular}{|c|c|c|c|c|c|c|c|c|}
\hline Code & Three way cross hybrids & $\begin{array}{l}\text { Days to } \\
\text { tasseling }\end{array}$ & $\begin{array}{l}\text { Days to } \\
\text { silking }\end{array}$ & ASI & $\begin{array}{l}\text { Plant } \\
\text { height } \\
\text { (cm) }\end{array}$ & $\begin{array}{c}\text { Ear } \\
\text { height } \\
\text { (cm) }\end{array}$ & $\begin{array}{c}\text { Ear } \\
\text { length } \\
\text { (cm) }\end{array}$ & $\begin{array}{c}\text { Ear } \\
\text { circumference } \\
(\mathrm{cm})\end{array}$ \\
\hline TWC 1 & $($ HKI-26-2-4-(1-2) × CML-41) $\times$ CML-439 & 57.37 & 59.92 & 1.58 & 223.72 & 120.60 & 17.26 & 15.02 \\
\hline TWC 2 & $($ HKI-26-2-4-(1-2) × CML-41) $\times$ DMRN-21 & 57.85 & 60.40 & 1.33 & 224.62 & 129.30 & 16.11 & 14.51 \\
\hline TWC 3 & $($ HKI-26-2-4-(1-2) × CML-41) × CML-139 & 57.21 & 59.59 & 1.35 & 206.81 & 116.03 & 15.90 & 14.14 \\
\hline TWC 4 & $($ CML-359 $\times$ CML-326) $\times$ CML-139 & 70.13 & 72.04 & 0.32 & 208.75 & 106.14 & 16.56 & 14.11 \\
\hline TWC 5 & $(\mathrm{CML}-359 \times$ CML-326) $\times$ DMRN-21 & 61.16 & 63.07 & 0.89 & 201.65 & 109.89 & 16.05 & 14.10 \\
\hline TWC 6 & $(\mathrm{CML}-359 \times$ CML-326) $\times$ CML-139 & 58.85 & 61.00 & 1.06 & 202.29 & 105.38 & 15.84 & 14.47 \\
\hline TWC 7 & $($ CML 470-Bx15 × CML-41) $\times$ CML-139 & 59.74 & 63.31 & 1.57 & 212.98 & 110.41 & 16.39 & 14.50 \\
\hline TWC 8 & $(\mathrm{CML} 470-\mathrm{Bx} 15 \times \mathrm{CML}-41) \times \mathrm{DMRN}-21$ & 60.39 & 62.91 & 1.06 & 209.46 & 122.98 & 16.40 & 14.58 \\
\hline TWC 9 & $(\mathrm{CML} 470-\mathrm{Bx} 15 \times \mathrm{CML}-41) \times \mathrm{CML}-139$ & 61.64 & 63.72 & 0.76 & 198.31 & 109.15 & 15.29 & 14.67 \\
\hline TWC 10 & $($ HKI-26-2-4-(1-2) × CML-358) × CML-139 & 61.33 & 63.52 & 0.79 & 210.06 & 112.19 & 16.56 & 14.64 \\
\hline TWC 11 & $(\mathrm{HKI}-26-2-4-(1-2) \times \mathrm{CML}-358) \times \mathrm{DMRN}-21$ & 61.51 & 62.75 & 0.50 & 212.50 & 112.85 & 16.86 & 14.02 \\
\hline TWC 12 & $($ HKI-26-2-4-(1-2) × CML-358) × CML-139 & 58.56 & 60.28 & 1.16 & 217.85 & 119.71 & 16.20 & 14.07 \\
\hline TWC 13 & $($ HKI-26-2-4-(1-2) × CM-500) × CML-139 & 59.61 & 61.29 & 1.06 & 202.08 & 102.94 & 16.23 & 14.59 \\
\hline TWC 14 & $($ HKI-26-2-4-(1-2) $\times$ CM-500) $\times$ DMRN-21 & 59.79 & 61.33 & 0.81 & 203.40 & 109.13 & 16.59 & 13.81 \\
\hline TWC 15 & $(\mathrm{HKI}-26-2-4-(1-2) \times \mathrm{CM}-500) \times \mathrm{CML}-139$ & 58.02 & 60.21 & 1.38 & 209.23 & 114.56 & 15.80 & 14.86 \\
\hline TWC 16 & $($ HKI-26-2-4-(1-2) × CML-411) × CML-139 & 58.05 & 60.58 & 1.36 & 211.92 & 113.33 & 16.21 & 14.93 \\
\hline TWC 17 & $(\mathrm{HKI}-26-2-4-(1-2) \times \mathrm{CML}-411) \times \mathrm{DMRN}-21$ & 58.71 & 60.96 & 1.24 & 203.35 & 115.13 & 16.19 & 14.39 \\
\hline TWC 18 & $($ HKI-26-2-4-(1-2) × CML-411) × CML-139 & 58.42 & 60.38 & 1.10 & 176.25 & 92.00 & 16.86 & 14.58 \\
\hline Check 1 & Nithyashree & 64.60 & 66.10 & 1.50 & 221.48 & 121.58 & 16.15 & 15.10 \\
\hline \multirow[t]{2}{*}{ Check 2} & Hema & 65.90 & 68.50 & 2.60 & 214.42 & 122.83 & 15.20 & 15.67 \\
\hline & S.Em \pm & 0.79 & 0.67 & 0.81 & 2.52 & 1.96 & 0.12 & 0.08 \\
\hline
\end{tabular}




\begin{tabular}{|c|c|c|c|c|c|c|}
\hline Code & Three way cross hybrids & $\begin{array}{l}\text { Kernels } \\
\text { row }^{-1}\end{array}$ & $\begin{array}{c}\text { Kernel } \\
\text { rows } \\
\text { ear }^{-1}\end{array}$ & $\begin{array}{c}100 \text { grain } \\
\text { weight } \\
\text { (g) }\end{array}$ & $\begin{array}{c}\text { Grain yield } \\
\text { plant }^{-1}(\mathrm{~g})\end{array}$ & $\begin{array}{c}\text { Shelling } \\
\text { percentage }\end{array}$ \\
\hline TWC 1 & $(\mathrm{HKI}-26-2-4-(1-2) \times$ CML-41) $\times$ CML-439 & 35.43 & 15.76 & 26.24 & 128.13 & 83.41 \\
\hline TWC 2 & $($ HKI-26-2-4-(1-2) $\times$ CML-41) $\times$ DMRN-21 & 33.98 & 15.39 & 26.67 & 126.24 & 85.64 \\
\hline TWC 3 & $($ HKI-26-2-4-(1-2) × CML-41) $\times$ CML-139 & 31.54 & 15.76 & 25.23 & 102.79 & 85.52 \\
\hline TWC 4 & $(\mathrm{CML}-359 \times$ CML-326) $\times$ CML-139 & 34.17 & 15.11 & 26.37 & 119.51 & 85.83 \\
\hline TWC 5 & $(\mathrm{CML}-359 \times \mathrm{CML}-326) \times \mathrm{DMRN}-21$ & 33.90 & 14.69 & 25.03 & 114.87 & 85.39 \\
\hline TWC 6 & $($ CML-359 $\times$ CML-326) $\times$ CML-139 & 33.22 & 14.67 & 26.47 & 117.46 & 85.62 \\
\hline TWC 7 & $(\mathrm{CML}$ 470-Bx15 × CML-41) $\times$ CML-139 & 30.49 & 15.36 & 27.14 & 107.08 & 89.70 \\
\hline TWC 8 & $(\mathrm{CML} 470-\mathrm{Bx} 15 \times \mathrm{CML}-41) \times \mathrm{DMRN}-21$ & 32.10 & 14.42 & 28.57 & 111.77 & 83.43 \\
\hline TWC 9 & $(\mathrm{CML} 470-\mathrm{Bx} 15 \times \mathrm{CML}-41) \times \mathrm{CML}-139$ & 29.06 & 15.01 & 28.65 & 110.75 & 83.72 \\
\hline TWC 10 & $($ HKI-26-2-4-(1-2) × CML-358) × CML-139 & 34.73 & 15.36 & 24.43 & 113.41 & 82.90 \\
\hline TWC 11 & $($ HKI-26-2-4-(1-2) × CML-358) × DMRN-21 & 34.03 & 14.99 & 25.34 & 114.65 & 85.20 \\
\hline TWC 12 & $(\mathrm{HKI}-26-2-4-(1-2) \times \mathrm{CML}-358) \times \mathrm{CML}-139$ & 31.37 & 15.33 & 25.06 & 105.25 & 84.25 \\
\hline TWC 13 & $(\mathrm{HKI}-26-2-4-(1-2) \times \mathrm{CM}-500) \times \mathrm{CML}-139$ & 34.07 & 14.54 & 25.16 & 106.12 & 83.17 \\
\hline TWC 14 & $($ HKI-26-2-4-(1-2) × CM-500) $\times$ DMRN-21 & 33.32 & 14.53 & 24.34 & 105.17 & 84.63 \\
\hline TWC 15 & $(\mathrm{HKI}-26-2-4-(1-2) \times \mathrm{CM}-500) \times \mathrm{CML}-139$ & 32.05 & 15.00 & 27.75 & 125.49 & 84.25 \\
\hline TWC 16 & $($ HKI-26-2-4-(1-2) × CML-411) × CML-139 & 35.40 & 15.00 & 25.73 & 121.92 & 83.08 \\
\hline TWC 17 & $($ HKI-26-2-4-(1-2) × CML-411) × DMRN-21 & 33.44 & 14.60 & 27.55 & 123.19 & 86.31 \\
\hline TWC 18 & $($ HKI-26-2-4-(1-2) × CML-411) × CML-139 & 33.26 & 14.31 & 29.11 & 119.00 & 83.97 \\
\hline Check 1 & Nithyashree & 29.90 & 15.40 & 27.67 & 126.00 & 81.44 \\
\hline \multirow[t]{2}{*}{ Check 2} & Hema & 31.43 & 16.13 & 26.49 & 119.00 & 81.42 \\
\hline & S.Em \pm & 0.40 & 0.10 & 0.35 & 1.88 & 0.38 \\
\hline
\end{tabular}


Table.3 Estimates of realized and predicted mean performances of 18 three way cross hybrids for 12 quantitative traits in maize

\begin{tabular}{|c|c|c|c|c|c|c|c|}
\hline \multirow[t]{2}{*}{ Code } & \multirow[t]{2}{*}{ Three way cross hybrids } & \multicolumn{3}{|c|}{ Days to tasseling } & \multicolumn{3}{|c|}{ Days to silking } \\
\hline & & Realized & Predicted & $\chi^{2}$ statistic & Realized & Predicted & $\chi^{2}$ statistic \\
\hline TWC 1 & $($ HKI-26-2-4-(1-2) × CML-41) $\times$ CML-439 & 57.37 & 62.95 & $789.80 * *$ & 59.92 & 64.45 & $471.25 * *$ \\
\hline TWC 2 & $($ HKI-26-2-4-(1-2) × CML-41) × DMRN-21 & 57.85 & 62.60 & $337.38 * *$ & 60.40 & 63.50 & $136.03 * *$ \\
\hline TWC 3 & $($ HKI-26-2-4-(1-2) × CML-41) $\times$ CML-139 & 57.21 & 61.90 & $991.56 * *$ & 59.59 & 62.78 & $424.58 * *$ \\
\hline TWC 4 & $($ CML-359 $\times$ CML-326) $\times$ CML-139 & 70.13 & 64.80 & $297.43 * *$ & 72.04 & 67.18 & $71.21 * *$ \\
\hline TWC 5 & $(\mathrm{CML}-359 \times \mathrm{CML}-326) \times \mathrm{DMRN}-21$ & 61.16 & 61.60 & 0.61 & 63.07 & 62.58 & 0.78 \\
\hline TWC 6 & $(\mathrm{CML}-359 \times$ CML-326) $\times$ CML-139 & 58.85 & 63.85 & $80.67 * *$ & 61.00 & 64.97 & $58.82 * *$ \\
\hline TWC 7 & $(\mathrm{CML} 470-\mathrm{Bx} 15 \times \mathrm{CML}-41) \times \mathrm{CML}-139$ & 59.74 & 62.75 & $63.23 * *$ & 63.31 & 64.52 & 7.37 \\
\hline TWC 8 & $(\mathrm{CML} 470-\mathrm{B} \times 15 \times$ CML-41) $\times$ DMRN-21 & 60.39 & 62.60 & $36.18^{*}$ & 62.91 & 63.82 & 3.18 \\
\hline TWC 9 & $($ CML 470-Bx15 × CML-41) $\times$ CML-139 & 61.64 & 60.60 & 6.28 & 63.72 & 60.93 & $45.36 * *$ \\
\hline TWC 10 & $($ HKI-26-2-4-(1-2) × CML-358) × CML-139 & 61.33 & 63.30 & $36.37 *$ & 63.52 & 64.55 & 8.81 \\
\hline TWC 11 & $($ HKI-26-2-4-(1-2) × CML-358) $\times$ DMRN-21 & 61.51 & 61.75 & 0.11 & 62.75 & 63.25 & 0.56 \\
\hline TWC 12 & $($ HKI-26-2-4-(1-2) × CML-358) × CML-139 & 58.56 & 62.65 & $260.68 * *$ & 60.28 & 63.80 & $196.93 * *$ \\
\hline TWC 13 & $($ HKI-26-2-4-(1-2) $\times$ CM-500) $\times$ CML-139 & 59.61 & 62.35 & $77.05 * *$ & 61.29 & 64.00 & $82.47 * *$ \\
\hline TWC 14 & $($ HKI-26-2-4-(1-2) × CM-500) × DMRN-21 & 59.79 & 61.70 & 25.27 & 61.33 & 62.85 & 21.53 \\
\hline TWC 15 & $($ HKI-26-2-4-(1-2) × CM-500) $\times$ CML-139 & 58.02 & 61.75 & $669.75 * *$ & 60.21 & 63.10 & $176.30 * *$ \\
\hline TWC 16 & $($ HKI-26-2-4-(1-2) × CML-411) × CML-139 & 58.05 & 62.05 & $197.35 * *$ & 60.58 & 63.50 & $132.74 * *$ \\
\hline TWC 17 & $($ HKI-26-2-4-(1-2) × CML-411) $\times$ DMRN-21 & 58.71 & 61.55 & $83.31 * *$ & 60.96 & 62.07 & 9.91 \\
\hline TWC 18 & (HKI-26-2-4-(1-2) × CML-411) × CML-139 & 58.42 & 62.20 & $200.43 * *$ & 60.38 & 63.05 & $67.38 * *$ \\
\hline
\end{tabular}

*Significant at $\mathrm{P} \leq 0.05,{ }^{*}$ Significant at $\mathrm{P} \leq 0.01, * * *$ Significant at $\mathrm{P} \leq 0.001$ 
Contd...

\begin{tabular}{|c|c|c|c|c|c|c|c|}
\hline \multirow[t]{2}{*}{ Code } & \multirow[t]{2}{*}{ Three way cross hybrids } & \multicolumn{3}{|c|}{ Ear height (cm) } & \multicolumn{3}{|c|}{ Ear length (cm) } \\
\hline & & Realized & Predicted & $\chi 2$ statistic & Realized & Predicted & $\chi^{2}$ statistic \\
\hline TWC 1 & (HKI-26-2-4-(1-2) × CML-41) × CML-439 & 120.60 & 107.32 & $50.45^{* *}$ & 17.26 & 16.44 & 33.77 \\
\hline TWC 2 & $($ HKI-26-2-4-(1-2) × CML-41) × DMRN-21 & 129.30 & 112.11 & $132.07 * *$ & 16.11 & 15.96 & 0.88 \\
\hline TWC 3 & (HKI-26-2-4-(1-2) × CML-41) × CML-139 & 116.03 & 113.89 & 0.55 & 15.90 & 15.57 & 3.87 \\
\hline TWC 4 & $(\mathrm{CML}-359 \times$ CML-326) $\times$ CML-139 & 106.14 & 113.99 & 16.28 & 16.56 & 14.72 & $151.06 * *$ \\
\hline TWC 5 & $(\mathrm{CML}-359 \times \mathrm{CML}-326) \times \mathrm{DMRN}-21$ & 109.89 & 117.10 & 8.27 & 16.05 & 17.04 & 29.79 \\
\hline TWC 6 & $(\mathrm{CML}-359 \times$ CML-326) $\times$ CML-139 & 105.38 & 112.38 & 18.86 & 15.84 & 14.73 & $58.55^{* *}$ \\
\hline TWC 7 & $(\mathrm{CML} 470-\mathrm{Bx} 15 \times \mathrm{CML}-41) \times \mathrm{CML}-139$ & 110.41 & 107.28 & 3.28 & 16.39 & 15.78 & 8.94 \\
\hline TWC 8 & $(\mathrm{CML} 470-\mathrm{B} \times 15 \times \mathrm{CML}-41) \times \mathrm{DMRN}-21$ & 122.98 & 117.72 & 6.37 & 16.40 & 15.55 & 19.44 \\
\hline TWC 9 & $(\mathrm{CML} 470-\mathrm{Bx} 15 \times \mathrm{CML}-41) \times \mathrm{CML}-139$ & 109.15 & 114.67 & 2.98 & 15.29 & 14.34 & 27.72 \\
\hline TWC 10 & $($ HKI-26-2-4-(1-2) × CML-358) × CML-139 & 112.19 & 108.61 & 1.47 & 16.56 & 16.50 & 0.16 \\
\hline TWC 11 & $(\mathrm{HKI}-26-2-4-(1-2) \times \mathrm{CML}-358) \times \mathrm{DMRN}-21$ & 112.85 & 112.20 & 0.04 & 16.86 & 16.90 & 0.05 \\
\hline TWC 12 & $($ HKI-26-2-4-(1-2) × CML-358) × CML-139 & 119.71 & 112.98 & 3.78 & 16.20 & 16.51 & 2.29 \\
\hline TWC 13 & $($ HKI-26-2-4-(1-2) × CM-500) × CML-139 & 102.94 & 102.98 & 0.0002 & 16.23 & 16.75 & 4.72 \\
\hline TWC 14 & $($ HKI-26-2-4-(1-2) $\times$ CM-500) $\times$ DMRN-21 & 109.13 & 104.45 & 2.18 & 16.59 & 16.97 & 3.86 \\
\hline TWC 15 & $($ HKI-26-2-4-(1-2) × CM-500) $\times$ CML-139 & 114.56 & 105.79 & 9.29 & 15.80 & 16.72 & 32.21 \\
\hline TWC 16 & $($ HKI-26-2-4-(1-2) × CML-411) × CML-139 & 113.33 & 111.71 & 0.26 & 16.21 & 16.19 & 0.02 \\
\hline TWC 17 & $($ HKI-26-2-4-(1-2) × CML-411) $\times$ DMRN-21 & 115.13 & 112.96 & 0.65 & 16.19 & 16.71 & 8.87 \\
\hline TWC 18 & $($ HKI-26-2-4-(1-2) × CML-411) × CML-139 & 92.00 & 115.66 & $109.58 * *$ & 16.86 & 16.23 & 12.89 \\
\hline
\end{tabular}

* Significant at $\mathrm{P} \leq 0.05$, ** Significant at $\mathrm{P} \leq 0.01$, *** Significant at $\mathrm{P} \leq 0.001$ 
Contd...

\begin{tabular}{|c|c|c|c|c|c|c|c|}
\hline \multirow[t]{2}{*}{ Code } & \multirow[t]{2}{*}{ Three way cross hybrids } & \multicolumn{3}{|c|}{ Ear circumference $(\mathrm{cm})$} & \multicolumn{3}{|c|}{ Kernels row ${ }^{-1}$} \\
\hline & & Realized & Predicted & $\chi^{2}$ statistic & Realized & Predicted & $\chi^{2}$ statistic \\
\hline TWC 1 & $($ HKI-26-2-4-(1-2) × CML-41) $\times$ CML-439 & 15.02 & 14.88 & 2.82 & 35.43 & 34.70 & 2.27 \\
\hline TWC 2 & $(\mathrm{HKI}-26-2-4-(1-2) \times \mathrm{CML}-41) \times \mathrm{DMRN}-21$ & 14.51 & 14.30 & 8.27 & 33.98 & 35.90 & 25.56 \\
\hline TWC 3 & $($ HKI-26-2-4-(1-2) × CML-41) $\times$ CML-139 & 14.14 & 14.57 & 32.59 & 31.54 & 33.13 & 11.46 \\
\hline TWC 4 & $(\mathrm{CML}-359 \times$ CML-326) $\times$ CML-139 & 14.11 & 13.42 & $121.10 * *$ & 34.17 & 31.45 & $34.84 *$ \\
\hline TWC 5 & $(\mathrm{CML}-359 \times \mathrm{CML}-326) \times \mathrm{DMRN}-21$ & 14.10 & 14.21 & 4.74 & 33.90 & 39.45 & $141.10 * *$ \\
\hline TWC 6 & $(\mathrm{CML}-359 \times$ CML-326) $\times$ CML-139 & 14.47 & 13.83 & $72.34 * *$ & 33.22 & 31.52 & 14.62 \\
\hline TWC 7 & $(\mathrm{CML} 470-\mathrm{Bx} 15 \times \mathrm{CML}-41) \times \mathrm{CML}-139$ & 14.50 & 15.18 & $132.56^{* *}$ & 30.49 & 33.25 & 21.45 \\
\hline TWC 8 & $(\mathrm{CML} 470-\mathrm{B} \times 15 \times \mathrm{CML}-41) \times \mathrm{DMRN}-21$ & 14.58 & 14.61 & 0.13 & 32.10 & 33.60 & 7.69 \\
\hline TWC 9 & $(\mathrm{CML} 470-\mathrm{Bx} 15 \times \mathrm{CML}-41) \times \mathrm{CML}-139$ & 14.67 & 14.44 & 6.34 & 29.06 & 29.80 & 4.06 \\
\hline TWC 10 & $(\mathrm{HKI}-26-2-4-(1-2) \times \mathrm{CML}-358) \times \mathrm{CML}-139$ & 14.64 & 13.72 & $217.30 * *$ & 34.73 & 34.53 & 0.15 \\
\hline TWC 11 & $($ HKI-26-2-4-(1-2) × CML-358) $\times$ DMRN-21 & 14.02 & 14.43 & 27.51 & 34.03 & 38.45 & $63.01 * *$ \\
\hline TWC 12 & $($ HKI-26-2-4-(1-2) × CML-358) × CML-139 & 14.07 & 14.32 & 21.41 & 31.37 & 35.85 & $82.00 * *$ \\
\hline TWC 13 & $(\mathrm{HKI}-26-2-4-(1-2) \times \mathrm{CM}-500) \times \mathrm{CML}-139$ & 14.59 & 14.39 & 10.93 & 34.07 & 36.75 & 15.90 \\
\hline TWC 14 & $(\mathrm{HKI}-26-2-4-(1-2) \times \mathrm{CM}-500) \times \mathrm{DMRN}-21$ & 13.81 & 14.13 & 19.86 & 33.32 & 36.95 & $63.09 * *$ \\
\hline TWC 15 & $($ HKI-26-2-4-(1-2) × CM-500) $\times$ CML-139 & 14.86 & 14.67 & 3.47 & 32.05 & 35.70 & $74.40 * *$ \\
\hline TWC 16 & $($ HKI-26-2-4-(1-2) × CML-411) × CML-139 & 14.93 & 14.46 & $44.94 * *$ & 35.40 & 35.72 & 0.39 \\
\hline TWC 17 & $($ HKI-26-2-4-(1-2) × CML-411) $\times$ DMRN-21 & 14.39 & 14.42 & 0.21 & 33.44 & 37.10 & 32.06 \\
\hline TWC 18 & $($ HKI-26-2-4-(1-2) × CML-411) × CML-139 & 14.58 & 14.46 & 1.39 & 33.26 & 34.02 & 5.00 \\
\hline
\end{tabular}

* Significant at $\mathrm{P} \leq 0.05, * *$ Significant at $\mathrm{P} \leq 0.01$, *** Significant at $\mathrm{P} \leq 0.001$ 
Contd...

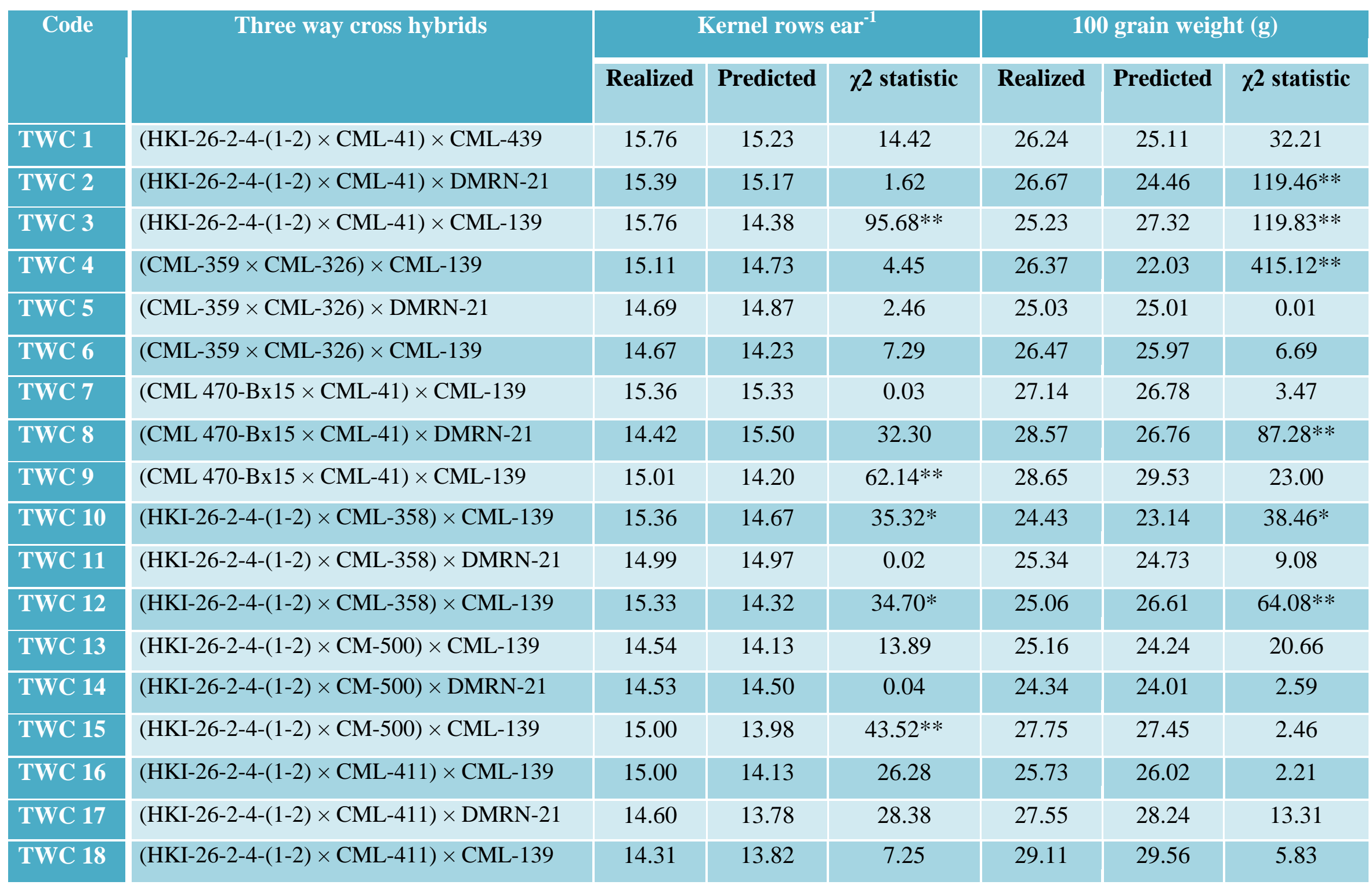

* Significant at $\mathrm{P} \leq 0.05,{ }^{* *}$ Significant at $\mathrm{P} \leq 0.01, * * *$ Significant at $\mathrm{P} \leq 0.001$ 
Contd...

\begin{tabular}{|c|c|c|c|c|c|c|c|}
\hline \multirow[t]{2}{*}{ Code } & \multirow[t]{2}{*}{ Three way cross hybrids } & \multicolumn{3}{|c|}{ Grain yield plant $^{-1}$} & \multicolumn{3}{|c|}{ Shelling percentage } \\
\hline & & Realized & Predicted & $\chi^{2}$ statistic & Realized & Predicted & $\chi^{2}$ statistic \\
\hline TWC 1 & $($ HKI-26-2-4-(1-2) × CML-41) × CML-439 & 128.13 & 115.17 & $19338.60 * *$ & 83.41 & 82.33 & $96.27 * *$ \\
\hline TWC 2 & $($ HKI-26-2-4-(1-2) × CML-41) × DMRN-21 & 126.24 & 123.67 & $819.99 * *$ & 85.64 & 86.89 & $136.05 * *$ \\
\hline TWC 3 & $($ HKI-26-2-4-(1-2) × CML-41) × CML-139 & 102.79 & 116.83 & $23032.53 * *$ & 85.52 & 83.38 & $381.96^{* *}$ \\
\hline TWC 4 & $($ CML-359 × CML-326) $\times$ CML-139 & 119.51 & 93.50 & $63273.55^{* *}$ & 85.83 & 84.35 & $184.05 * *$ \\
\hline TWC 5 & $($ CML-359 $\times$ CML-326) $\times$ DMRN-21 & 114.87 & 126.00 & $15603.46 * *$ & 85.39 & 86.27 & $66.60 * *$ \\
\hline TWC 6 & $($ CML-359 $\times$ CML-326) $\times$ CML-139 & 117.46 & 106.67 & $12427.97 * *$ & 85.62 & 85.83 & 3.56 \\
\hline TWC 7 & $(\mathrm{CML} 470-\mathrm{Bx} 15 \times \mathrm{CML}-41) \times \mathrm{CML}-139$ & 107.08 & 119.00 & $16898.83 * *$ & 89.70 & 82.45 & $4334.51 * *$ \\
\hline TWC 8 & $($ CML 470-Bx15 × CML-41) $\times$ DMRN-21 & 111.77 & 130.50 & $45763.95 * *$ & 83.43 & 87.33 & $1329.29 * *$ \\
\hline TWC 9 & $($ CML 470-Bx15 × CML-41) $\times$ CML-139 & 110.75 & 112.33 & $281.61 * *$ & 83.72 & 84.24 & 23.09 \\
\hline TWC 10 & $($ HKI-26-2-4-(1-2) × CML-358) × CML-139 & 113.41 & 104.67 & $8004.70 * *$ & 82.90 & 83.17 & 6.31 \\
\hline TWC 11 & $($ HKI-26-2-4-(1-2) $\times$ CML-358) $\times$ DMRN-21 & 114.65 & 132.83 & $43935.50 * *$ & 85.20 & 85.89 & $40.94 *$ \\
\hline TWC 12 & $($ HKI-26-2-4-(1-2) × CML-358) × CML-139 & 105.25 & 119.83 & $25501.14 * *$ & 84.25 & 82.41 & $278.75^{* *}$ \\
\hline TWC 13 & $($ HKI-26-2-4-(1-2) × CM-500) × CML-139 & 106.12 & 112.67 & $4823.25 * *$ & 83.17 & 82.33 & $57.63 * *$ \\
\hline TWC 14 & $(\mathrm{HKI}-26-2-4-(1-2) \times \mathrm{CM}-500) \times \mathrm{DMRN}-21$ & 105.17 & 123.17 & $39911.47 * *$ & 84.63 & 86.09 & $182.36 * *$ \\
\hline TWC 15 & $($ HKI-26-2-4-(1-2) × CM-500) × CML-139 & 125.49 & 122.67 & $975.11 * *$ & 84.25 & 82.85 & $163.18 * *$ \\
\hline TWC 16 & $($ HKI-26-2-4-(1-2) × CML-411) × CML-139 & 121.92 & 119.67 & $608.81 * *$ & 83.08 & 83.28 & 3.54 \\
\hline TWC 17 & $($ HKI-26-2-4-(1-2) $\times$ CML-411) $\times$ DMRN-21 & 123.19 & 134.33 & $16688.00 * *$ & 86.31 & 85.93 & 12.63 \\
\hline TWC 18 & $($ HKI-26-2-4-(1-2) × CML-411) × CML-139 & 119.00 & 128.83 & $12469.92 * *$ & 83.97 & 84.19 & 3.98 \\
\hline
\end{tabular}

* Significant at $\mathrm{P} \leq 0.05,{ }^{* *}$ Significant at $\mathrm{P} \leq 0.01, * * *$ Significant at $\mathrm{P} \leq 0.001$ 
Good agreement between realised and predicted performances of three way crosses indicating the adequacy of additivedominance model in the inheritance of all characters except grain yield plant $^{-1}$ and shelling percentage (Table 3 ). The rationale of high predictive power of the prediction method is that for any individual locus, the three way cross hybrids $\{(\mathrm{A} \times \mathrm{B}) \times \mathrm{C}\}$ includes only those genotypes which are produced in the AC and BC single crosses. Thus, the magnitude of additive and dominance effects expressed in three way cross hybrids would be the same as that of non-parental single cross hybrids. These two populations i.e., 'three way crosses' and a group of non-parental single crosses however, may differ with respect to a few specific combinations of genes at different loci which is of course inconsequential so long as genes at different loci are independent in action, i.e., epistasis is absent (Hallauer and Miranda, 1988). In prediction method, all 18 three way crosses manifested significant differences between realised and predicted performance suggesting involvement of epistasis in genetic control of grain yield plant ${ }^{-1}$ (Bauman 1959, Chahal and Gosal, 2002) (Table 3). By and large, there was a good agreement between realized and predicted performance of three way cross hybrids for all characters except for grain yield plant ${ }^{-1}$ and shelling percentage.

\section{References}

Bauman, L. F., 1959. Evident of non-allelic gene interaction in determining yield, and kernel row number in corn. Agron. J., 31: 531-534.

Chahal, G. S. and Gosal, S. S., 2002. Principles and procedures of plant breeding. Narosa Publishing House, New Delhi, India, 604pp.

Crabb, R. A. 1992. The hybrid corn makers. New Brunswick, N. J. Rutgers Univ. press p 331.

Hallauer, A. R. and Miranda Filho, J. B., 1988. Quantitative genetics in maize breeding. Iowa State Univ. press, Ames, Iwoa, UAS.

Jenkins, M. T., 1934. Methods of estimating the performance of double crosses in corn. J. Amer. Soc. Agron., 26: 199-204. Jones (1918)

Shull, G. H., 1908. The composition of a field of maize. American Breeders Association Report, 4: 296-301.

Shull, G. H., 1911. The genotypes of maize. American Naturalist, 45: 234-252.

\section{How to cite this article:}

Pavan, R., E. Gangappa, S. Ramesh, A. Mohan Rao and Shailaja Hittalmani. 2018. Prediction and Validation of Three Cross Hybrids in Maize (Zea mays L.). Int.J.Curr.Microbiol.App.Sci. 7(01): 1502-1512. doi: https://doi.org/10.20546/ijcmas.2018.701.183 